[0212-7199 (2003) 20: 6; pp 287-291] ANALES DE MEDICINA INTERNA Copyright @ 2003 ARAN EDICIONES, S.L.

AN. MED. INTERNA (Madrid) Vol. 20, N. ${ }^{\circ} 6$, pp. $287-291,2003$

\section{Valoración de la semiología psicosomática en hipertensos tratados con lercanidipino (estudio LERCAPSICO)}

\author{
J. ABELLÁN ALEMÁN, J. F. MARTÍNEZ GARCÍA, J. MERINO SÁNCHEZ, V. GIL \\ GUILLÉN, J. LATORRE HERNÁNDEZ, J. A. DIVISÓN GARROTE, C. FERNÁNDEZ \\ TORRES, F. FERNÁNDEZ MONTERO, A. NAVARRO LIMA, EN \\ REPRESENTACIÓN DEL GRUPO DE INVESTIGADORES DEL ESTUDIO \\ LERCAPSICO
}

Centros de Atención Primaria de Murcia, Alicante, Almería, Albacete, Granada y Jaén

EVALUATION OF PSICOSOMATIC SEMIOLOGY IN HYPERTENSIVE PATIENTS TREATED WITH LECARNIDIPINE (LERCAPSICO STUDY)

\section{RESUMEN}

Objetivo: Valorar el grado de ansiedad y semiología psicosomática en hipertensos tratados con lercanidipino y analizar su evolución. Secundariamente se plantea un estudio de farmacovigilancia con lercanidipino.

Material y métodos: Se diseña un estudio prospectivo observacional multicéntrico, de 6 meses de seguimiento para hipertensos esenciales con PAS entre 140-180 mm Hg y/o 90-110 mm Hg de PAD. Tras un periodo de lavado de 10 días, se inicia tratamiento con lercanidipino $10 \mathrm{mg}$ (1-0$0)$. Si no se logra control de la PA se asocia ramipril $2,5 \mathrm{mg} / \mathrm{día}$. Se realizan controles clínicos periódicos con medición de la PA, FC, valoración objetiva de tolerancia y observancia. Al inicio y al final del estudio se practican controles bioquímicos y se valora el nivel de ansiedad (cuestionario STAI; subescala Rasgo) (Valoración en decatipos 0-4 ausencia de ansiedad 4-7 ansiedad moderada 7-10 ansiedad marcada) y el perfil psicosomático (cuestionario de diseño propio) (Escala 0-18; 0 mucha presencia de semiología, 18 total ausencia). La tolerancia clínica al fármaco se valora subjetiva y objetivamente.

Resultados: Se incluyen 538 pacientes. Se registran 54 abandonos. Por efectos secundarios el 3,75\%. Finalizan 484 (237 V, 247 M) de ellos 429 con lercanidipino en monoterapia $(88,6 \%)$. Edad media $60,9+10,7$ años. Su IMC fue de $29,1+5$. El grado de ansiedad no se alteró durante el estudio, pasó de 4,6+1,7 al inicio a 4,5 + 1,7 al final (valoración en decatipos) (ns) y la semiología psicosomática evolucionó favorablemente de 10,7 + 4,2 a 12,5 + 3,7 ( $<<0,00005)$. Por sexos la evolución es similar. La PAS media bajó de $165,6+12,2 \mathrm{~mm} \mathrm{Hg}$ a $137,9+10,4 \mathrm{~mm} \mathrm{Hg}$ $(\mathrm{p}<0,00005)$ y la PAD media disminuyó de $96,5+8,1 \mathrm{~mm} \mathrm{Hg}$ a $81,0+$ $6,1 \mathrm{~mm} \mathrm{Hg}(\mathrm{p}<0,00005)$. La tolerancia clínica fue muy buena. Los parámetros bioquímicos mejoraron significativamente: colesterolemia inicial $227,7 \mathrm{mg} / \mathrm{dl}$ y final $213,6 \mathrm{mg} / \mathrm{dl}$ ( $\mathrm{p}<0,00005$ ); glucosa inicial $108,4 \mathrm{mg} / \mathrm{dl}$ $\mathrm{y}$ final $105,7 \mathrm{mg} / \mathrm{dl}(\mathrm{p}<0,00005)$.

Conclusiones: Se comprueba un nivel medio de ansiedad en el colectivo analizado que no varía a lo largo del estudio. La semiología psicosomática se reduce significativamente. El lercanidipino se muestra muy eficaz en el control tensional y con una buena tolerancia.

PALABRAS CLAVE: Lercanidipino. Calidad de vida. Hipertensión arterial.

\section{ABSTRACT}

Objective: To value the grade of anxiety and psychosomatic semiology in hypertensive patients treated with lercanidipine and to analyse their evolution. A secondary objective is to carry out a pharmacovigilance study with lercanidipine.

Material and methods: Prospective multicentre observational 6 month study in primary hypertensive patients with SBP between 140-180 $\mathrm{mm} \mathrm{Hg}$ and/or 90-110 mm Hg DBP. After a washout period of 10 days, treatment with $10 \mathrm{mg}$ (1-0-0) lercanidipine is initiated. If BP is not controlled treatment with ramipril $2.5 \mathrm{mg} /$ day is instaured. Clinical checkups are carried periodically with measurements of BP. Heart Rate, objective valuation of tolerance to the drug and observance. At the initiation and end of the study biochemical check-ups are carried out, the level of anxiety is measured using the STAI questionnaire (Trait subscale) (Evaluation in decatipes 0-4 without ansiety 4-7 moderate ansiety, 710 high ansiety) and the psychosomatic profile with a questionnaire designed by this group. (Scale 0-18; 0 large semiology, 18 without semiology). Clinical tolerance to the drug is valued both subjectively and objectively.

Results: On included 538 patients. On registred 54 drop out, with side effects (3.75\%). Completed the study 484 (237 M, 247 F), 429 of them with Lercanidipine in monotherapy (88.6\%). Mean age $60.9 \pm$ 10.7. Mean BMI $29.1 \pm 5$. The grade of anxiety did not alter during the study passing from $4.6 \pm 1.7$ at the beginning of the study to $4.5 \pm 1.7$ at the end of the study (valuation in decatypes) (ns). The psychosomatic semiology changed favourably from $10.7 \pm 4.2$ to $12.5 \pm 3.7$ $(p<0.00005)$. The evolution according to sex is similar. The mean SBP decreased from $165.6 \pm 12.2 \mathrm{~mm} \mathrm{Hg}$ to $137.9 \pm 10.4 \mathrm{~mm} \mathrm{Hg}$ $(p<0.00005)$ and the mean DBP decreased from $96.5 \pm 8.1 \mathrm{~mm} \mathrm{Hg}$ to $81.0 \pm 6.1 \mathrm{~mm} \mathrm{Hg}(p<0.00005)$. Clinical tolerance was very good. Biochemical parameters were modified substantially: initial cholesterolemia $227.7 \mathrm{mg} / \mathrm{dl}$ and final cholesterolemia $213.6 \mathrm{mg} / \mathrm{dl}$ ( $p<0.00005)$; initial glucose $108.4 \mathrm{mg} / \mathrm{dl}$ and final glucose $105.7 \mathrm{mg} / \mathrm{dl}$ ( $p<0.00005)$.

Conclusions: The mean level of anxiety in the group studied is confirmed not to vary during the length of the study. Psychosomatic semiology is reduced being statistically significant. Lercanidipine is shown to be very effective as antihypertensive and well tolerated.

KEY WORDS: Lercanidipine. Quality of life. Blood pressure.

Abellán Alemán J, Martínez García JF, Merino Sánchez J, Gil Guillén V, Latorre Hernández J, Divisón Garrote JA, Fernández Torres C, Fernández. Montero F, Navarro Lima A, en representación del grupo de investigadores del Estudio Lercapsico. Valoración de la semiología psicosomática en hipertensos tratados con lercanidipino (estudio LERCAPSICO). An Med Interna (Madrid) 2003; 20: $287-291$. 


\section{INTRODUCCIÓN}

El VI informe del JNC (1) en las consideraciones sobre el tratamiento farmacológico de la Hipertensión Arterial dedica un apartado a la calidad de vida, reconoce que aunque algunos tratamientos antihipertensivos puedan causar efectos adversos, la calidad de vida se mantiene y probablemente mejora gracias a determinados fármacos recomendados. Aconseja estratificar los resultados en el tratamiento de la hipertensión en tres niveles: a corto, medio y largo plazo, los objetivos a corto plazo, los más relevantes para el médico clínico, incluyen medidas como el grado de control de la tensión arterial, el control bioquímico y la estabilidad o mejoría de los niveles de calidad de vida.

Hay acuerdo entre los investigadores en cuanto a la naturaleza multidimensional del concepto de calidad de vida relacionada con la salud (CVRS) (2-5), y abarca los tres aspectos de la vida del hombre: física, emocional y social. Ortega y Gasset (6) veía la vida como un proyecto a realizar y la enfermedad como un "algo" que impedía o entorpecía esa misión, para Julián Marías (7) la enfermedad es lo que dificulta, entorpece o imposibilita los proyectos, no meramente las funciones. La calidad subjetiva de vida es un estado de bienestar y de capacidad de actuación tal que permitiría la consecución de los objetivos personales por medio de las propias acciones y por tanto obtener la satisfacción derivada de ello.

La medida de la CVRS se realiza mediante escalas más o menos aceptadas que tratan de valorar los tres aspectos de la salud (8-10). Si bien todavía siguen siendo poco aplicados en la práctica clínica ya que están condicionados fundamentalmente por la extensión, posibilidad de autoadministración al paciente, facilidad de utilización y percepción por parte de los clínicos de la utilidad y relevancia de estas medidas (hay test que proporcionan mediciones muy precisas, pero que son extensos y complejos en su interpretación) (11-13).

Una de las acciones más importantes de los fármacos antihipertensivos además de garantizar una buena eficacia es mantener un tolerancia clínica que no perturbe la calidad de vida de los pacientes, área importante ya que en gran medida va unida a una buena observancia del tratamiento (14-22). Los calcioantagonistas son un grupo farmacológico heterogéneo y presentan una tolerancia clínica variable. Los calcioantagonistas preferentemente los dihidropiridínicos (Ca Dhp) promueven más semiología vasomotora resultando en una proporción mayor peor tolerados que el resto de los grupos de antihipertensivos (1).

Dadas las limitaciones que el empleo de los Ca Dhp de primera generación planteaban, se diseñaron otros de liberación más prolongada, lo que mejora su tolerancia (23). Recientes evidencias de estudios de intervención comunican una mejor tolerancia de estos fármacos y además una reducción de las complicaciones cardiovasculares con el empleo de estos Ca Dhp. $(1,24,25)$.

Por todo ello pareció interesante realizar un estudio de farmacovigilancia con Lercanidipino (Ca Dhp de última generación) en el cual además de la eficacia clínica y tolerancias clínica y bioquímica, se evaluara el nivel de ansiedad y la semiología psicosomática de los pacientes estudiados.

\section{MATERIAL Y MÉTODOS}

Se diseña un estudio clínico prospectivo, observacional abierto, no comparativo, multicéntrico realizado en Equipos de Atención Primaria de las provincias del Sureste peninsular: Murcia, Alicante, Albacete, Almería, Granada y Jaén, con un seguimiento a medio plazo (seis meses).

Se incluyen hipertensos esenciales de ambos sexos, entre 18 y 80 años de edad, en estadios 1 y 2 del Joint National Committee (cifras de PAS entre 140 y $180 \mathrm{mmHg}$ y/o PAD entre 90 y $110 \mathrm{mmHg}$ ). Y se excluyen los pacientes con HTA secundaria, angor inestable, IAM reciente (menos de seis meses), arritmias, ACV previo, insuficiencia hepática o renal, colagenopatías, inmunosupresión, hipersensibilidad a los calcioantagonistas, mujeres en edad fértil sin anticoncepción oral y los pacientes en tratamiento con fármacos que modifiquen la PA. Si los pacientes no eran de diagnóstico reciente y estaban previamente tratados con otros antihipertensivos se procedía a la retirada del tratamiento antihipertensivo y se seguía un periodo de lavado de 15 días bajo vigilancia médica, trancurrido éste y manteniéndose las condiciones de inclusión/exclusión se procedía a iniciar el estudio.

El diseño del estudio (Tabla I) comprendió una visita basal de captación donde se seleccionaba al paciente que cumplía los criterios de inclusión / exclusión, y cinco visitas más que detallamos a continuación:

- Visita inicial: donde el médico recogía la sintomatología clínica del paciente, se realizaba el control tensional, se solicitaba una analítica general y se realizaba al paciente el Cuestionario de Ansiedad STAI, escala Rasgo, así como un test de valoración psicosomática.

- Visita al primero, segundo y cuarto meses de seguimiento, donde se le realizaba controles clínicos y valoración de la tolerancia clínica al tratamiento.

-Visita final al sexto mes donde se le repetían todos los controles de la visita inicial.

\section{TABLA I}

\begin{tabular}{|c|c|c|c|c|c|c|}
\hline \multicolumn{7}{|c|}{ DISEÑO DEL ESTUDIO } \\
\hline & - 10 días & Inicial & $1^{\text {er }}$ Mes & 20 Mes & 40 Mes & 60 Mes \\
\hline Captación & $X$ & & & & & \\
\hline Clínica & & $x$ & $X$ & $X$ & $X$ & $X$ \\
\hline Analítica & & $x$ & & & & $X$ \\
\hline STAI (Rasgo) & & $x$ & & & & $x$ \\
\hline $\begin{array}{l}\text { Valoración } \\
\text { psicosomática }\end{array}$ & & $x$ & & & & $x$ \\
\hline
\end{tabular}

Para homogeneizar las determinaciones de la presión arterial, éstas se efectuaron con esfigmomanómetros automáticos OMRON, modelo $705 \mathrm{CP}$, siguiendo las recomendaciones del Consenso Español para el control de la HTA (26).

Para evaluar la ansiedad se utilizó el cuestionario STAI (subescala rasgo) (27). Esta escala consta de 20 ítem que se contestan en cuatro grados de gravedad, es sensible y fácil de completar y nos evalúa la tendencia a la ansiedad psicológica, no las alteraciones somáticas. Se considera que no presenta ansiedad si los valores obtenidos están entre 0-4, rasgos moderados de ansiedad si lo están entre 4-7 y ansiedad muy marcada cuando están entre 7-10 (valorada en decatipos). Además se realizó un cuestionario de valoración subjetiva de semiología psicosomática de 18 ítem de diseño propio y vali- 
dado (28). Escala de 0-18, en donde puntuaciones bajas reflejarían percepción de semiología psicosomática y las altas ausencia de la misma.

A todos los pacientes se les prescribió Lercanidipino a dosis de $10 \mathrm{mg}$ en toma única por las mañanas y a partir del segundo mes si no se había conseguido obtener cifras de normotensión se le asoció Ramipril a dosis de 2,5 mg también en toma única matutina.

El método estadístico realizado se ha basado en una estadística descriptiva de las variables analizadas con la obtención de la distribución de frecuencias absolutas y relativas y, en el caso de variables cuantitativas, se calcularon los parámetros característicos: media, mediana, rango, desviación típica. La evolución de los valores a lo largo del tiempo se realizó mediante un análisis de varianza de medias repetidas correspondiente a un diseño factorial jerarquizado teniendo en cuenta como factor principal el sexo y los pacientes como factor secundario (anidado) dentro del factor sexo.

\section{RESULTADOS}

Se incluyeron en el estudio 538 pacientes de los cuales completaron el estudio $484(89,9 \%)$. Se registraron 54 abandonos $(10,03 \%)$ (Fig. 1), de los que el 1,48\% lo fue por ineficacia, el $1,48 \%$ por incumplimiento terapéutico, el 3,02\% por propia iniciativa y el $3,75 \%$ por efectos secundarios, entre estos los que destacan los edemas maleolares y la rubefacción.

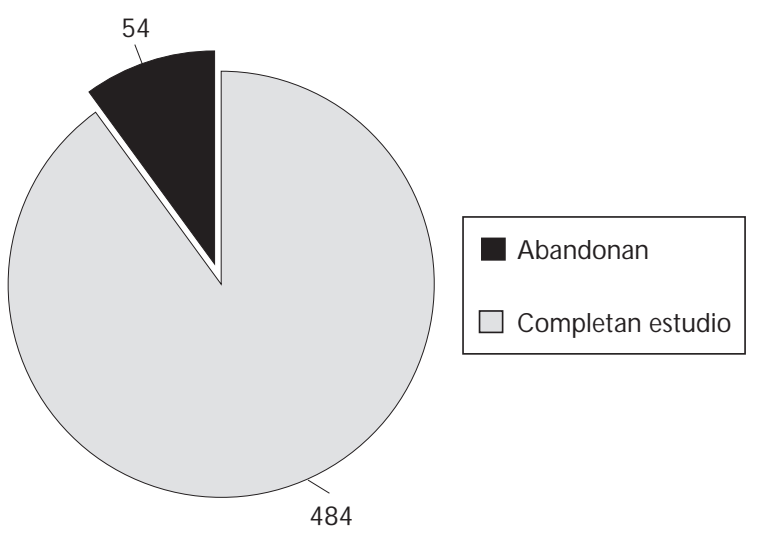

Fig. 1. Abandonos.

Las características de la muestra que completó el estudio fueron las siguientes: 247 mujeres (51\%) y 237 varones (49\%). La edad media fue de $60,9+10,7$ años. El peso medio inicial fue de $75,6+11.6 \mathrm{Kg}$, lo que corresponde a un IMC medio de 29,1 +5. El peso medio final fue de 75,3+11,3 Kg. ( $\mathrm{p}<0,0005)$.

Control tensional: el 88,7\% de los hipertensos se controlaron en monoterapia y el 11,3\% precisó asociarle ramipril en algún momento del seguimiento para el control tensional.

La PAS descendió desde una PAS media de 165,6 + 12,5 $\mathrm{mmHg}$ al inicio hasta $137,9+10,4 \mathrm{mmHg}$ al finalizar $(\mathrm{p}<$ 0,00005) (Fig. 2).

La PAD también descendió desde 96,5 + 8,1 mmHg a $81+$ $6,1 \mathrm{mmHg}$ durante el estudio ( $\mathrm{p}<0,00005$ ) (Fig. 2).
Grado de ansiedad, la puntuación inicial media del STAI fue de 4,6 + 1,7 y la final de 4,5 + 1,7 (n.s.), valorado en decatipos.

Valoración psicosomática el valor promedio inicial calculado fue de 10,7+4,2 y el final de 12,5+3,7 ( $<<0,00005)$.

La frecuencia cardiaca media evolucionó desde 80,5 a 76 ppm $(\mathrm{p}<0,00005)$.

La tolerancia clínica fue buena. Además de los abandonos (10\%), se encontraron efectos secundarios en el 3,76\% de los casos, siendo preferentemente derivados de la vasodilatación periférica: edemas maleolares y rubefacción facial y más frecuentes en el primer mes, disminuyendo posteriormente.

La tolerancia bioquímica muestra cifras de glucemia inicial media de 108,4 mg/dl y final de $105,7 \mathrm{mg} / \mathrm{dl}$ ( $\mathrm{p}<$ $0,00005)$. Colesterol inicial medio de $222,7 \mathrm{mg} / \mathrm{dl}$ y final de 213,6 mg/dl $(\mathrm{p}<0,00005)$. Colesterol HDL inicial medio de $50,9 \mathrm{mg} / \mathrm{dl}$ y final de $52,0 \mathrm{mg} / \mathrm{dl}$. ( $\mathrm{p}<0,003)$. Triglicéridos inicial medio de 152,9 mg/dl y final de $147,8 \mathrm{mg} / \mathrm{dl}$ $(\mathrm{p}<0,00001)$. Y Creatinina inicial media de $0,93 \mathrm{mg} / \mathrm{dl}$ y final de $0,92 \mathrm{mg} / \mathrm{dl}$ (n.s.).

\section{DISCUSIÓN}

Una característica importante del diseño de este estudio es su amplio tamaño muestral, 538 pacientes, lo que le confiere una alta potencia estadística al mismo.

Se ha encontrado una distribución por sexos muy ponderada, el $51 \%$ mujeres y el $49 \%$ varones, lo que no refleja la proporción real en la práctica clínica donde es mayor el porcentaje de mujeres que acuden a diario a nuestros centros de salud, probablemente porque (en especial en el medio rural) todavía tienen una mayor disponibilidad de horario, sin embargo la media de edad alta de la muestra $60,9+10,7$ años hace que se neutralice este efecto y el estudio refleja la realidad en cuanto a prevalencia de la hipertensión, una distribución similar de hipertensos entre hombres y mujeres en términos absolutos $(1,29)$.

$\mathrm{El}$ peso y el IMC medios de la muestra $(75,6+11,6 \mathrm{Kg}$ y un IMC 29,1 + 5) evidencian el sobrepeso de nuestra población general y en especial de los pacientes con algún factor de riesgo de enfermedades cardiovasculares. La evolución del peso a lo largo del estudio muestra una tendencia a disminuir con resultados significativos lo que ayuda a explicar en parte la buena evolución de los parámetros bioquímicos.

Recientemente ha cobrado importancia el control de la PAS, sobre todo porque es la tensión más difícil de normalizar $(30,31)$. Es habitual que la mayoría de los estudios de intervención acaban con la PAD controlada pero sin embargo la PAS está por encima de $140 \mathrm{mmHg}$. Las cifras finales del estudio (PAS final media 137,9 + 10,4 mmHg) son bastante elocuentes de la eficacia del fármaco empleado. La reducción es gradual y sostenida, a partir del primer mes se consigue que la PAD baje de $90 \mathrm{mmHg}$ y en torno al tercero y cuarto mes la PAS desciende por debajo de $140 \mathrm{mmHg}$. Estas cifras siguen un leve descenso hasta el final del estudio. Se comprueba pues el efecto beneficioso del Lercanidipino a dosis de $10 \mathrm{mg} . /$ día sobre la presión arterial, controlando en monoterapia el 88,7\% de los hipertensos incluidos, obteniendo reducciones de la PAS y PAD significativas.

Se verifica la buena tolerancia clínica del Lercanidipino, con la aparición de mínimos efectos secundarios, un 3,76\%, siendo éstos los típicos de los calcioantagonistas: edemas 


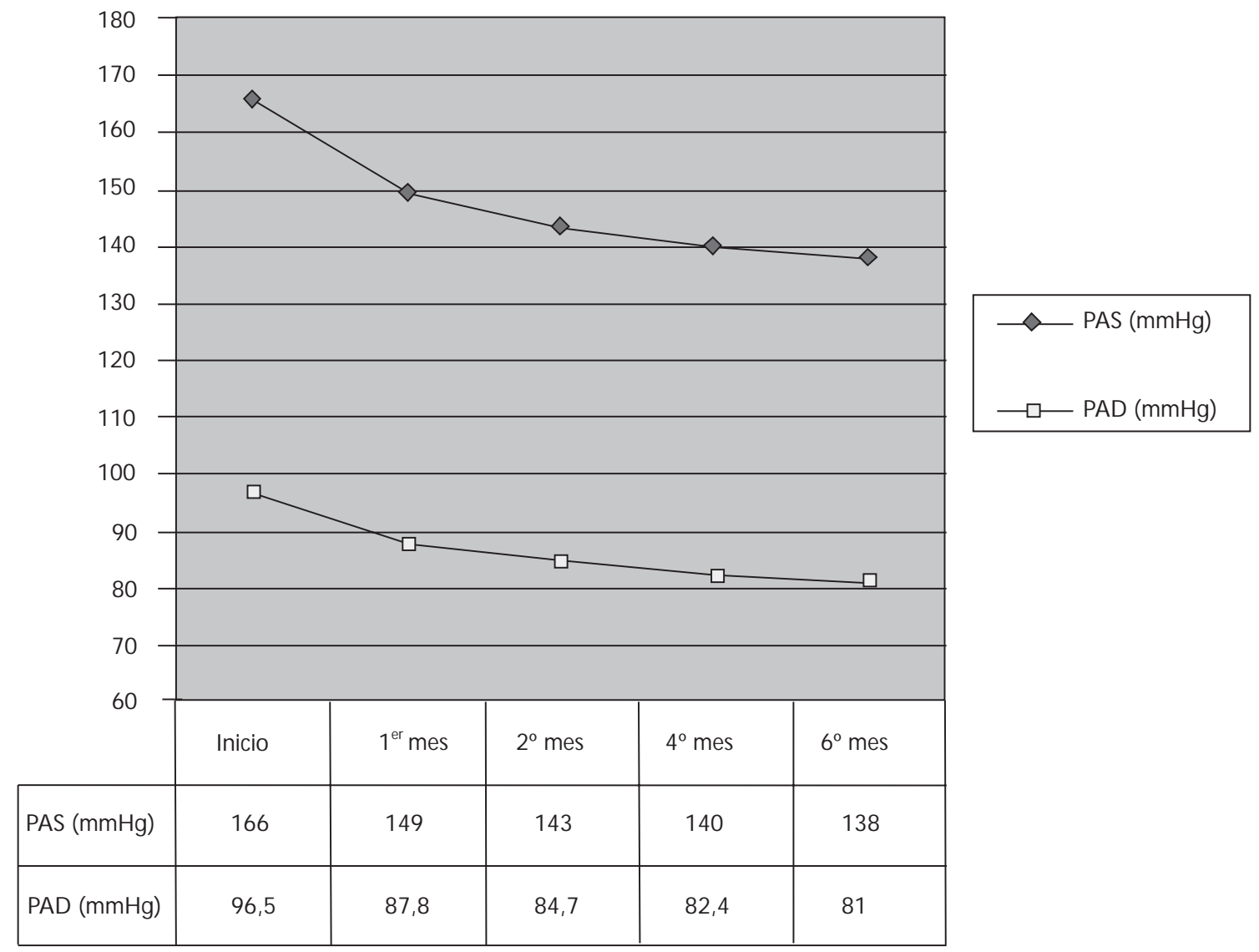

Fig. 2. Evolución de la presión arterial.

maleolares y rubefacción facial. Si bien puede parecer un bajo porcentaje de efectos secundarios debemos recordar que el Lercanidipino es un calcioantagonista con mínimos secundarismos $(21,23)$.

Múltiples estudios confirman que los diuréticos pueden aumentar los niveles de lípidos así como ocasionar una intolerancia a los hidratos de carbono; los betabloqueantes pueden aumentar los niveles de triglicéridos; los Iecas y los Calcioantagonistas son neutros en cuanto al perfil metabólico (32-35). En el estudio no sólo se verifican estos resultados sino que se mejora el perfil bioquímico de la muestra, se obtienen leves descensos de la glucosa, colesterol total, colesterol-HDL y triglicéridos que alcanzan niveles de significación estadística por el elevado número de la muestra.

Afortunadamente las opciones terapéuticas para el tratamiento de la HTA son numerosas, pero deberíamos disponer de más información sobre la influencia de estos fármacos sobre la calidad de vida de los pacientes (36), máxime cuando está demostrado que el cumplimiento terapéutico condiciona el éxito de la terapia antihipertensiva, que depende de la aceptación del paciente.

Existen evidencias que indican que la percepción de semiología, por parte del paciente, altera su calidad de vida y altera también la observancia terapéutica, lo que conlleva a un mal control tensional y éste otra vez a la alteración de la cali- dad de vida. Además muchas veces no se tiene en cuenta el grado de ansiedad de los pacientes, que bien por su predisposición previa, por el diagnóstico de enfermedad crónica, por la modificación de la conducta alimentaria y hábitos de vida, puede magnificar los efectos secundarios del tratamiento antihipertensivo (28).

En este estudio se comprueba un grado medio de ansiedad entre la población de hipertensos estudiados y que no varía a lo largo del mismo. El tratamiento con Lercanidipino no modifica la dimensión psicológica de la calidad de vida. Por el contrario, la semiología psicosomática, que hace referencia a los efectos adversos, sí sufre una reducción significativa estadísticamente durante el seguimiento.

La mejoría de la semiología psicosomática que se detecta podría atribuirse al uso del lercanidipino. Sin embargo, las características del diseño del estudio limitan la interpretación de tal acción al no disponer de un grupo control. La mejoría también podría deberse al mejor control tensional alcanzado, o a la mejor atención recibida por parte de los médicos que vigilaban la evolución. Sin embargo, cabe resaltar el dato objetivo de que dicha mejoría se constata en un grupo tratado con un calcioantagonista dihidropiridínico que es el grupo farmacológico que más perturba la tolerancia farmacológica de los hipertensos. Este dato realza la buena tolerancia que el lercanidipino tiene entre los hipertensos. 


\section{Bibliografía}

1. The Joint National Committee on Prevention, Detection, Evaluation and Treatment of High Blood Pressure, and the National Education Program Coordinating Committee. The sixth Report of the Joint National Committee on Prevention, Detection, Evaluation and Treatment of High Blood Pressure. Arch Intern Med 1997; 157: 2413-46.

2. Fernández JA, Orozco D, Hernández R. Calidad de vida relacionada con la salud: Una técnica comunicativa aplicable a la Atención Primaria. En: Manuales de referencia en Atención Primaria. Doyma 2000.

3. Oleson M. Subjective perceived quality of life. Image 1990; 22: 187-90.

4. Hernández-Mejía R, Fernández-López JA, Rancaño-García I. Epidemiología y Calidad de vida. Comparación entre variables sociodemográficas. En: V. Gil (Ed): Investigación y práctica clínica. Universidad Miguel Hernández. Alicante, 1999.

5. Fernández-López JA. Estudios de calidad de vida en Atención Primaria. En: Biblioteca Básica Dupont Pharma para médicos de Atención Primaria (VI); 1999: 69-89.

6. Ortega y Gasset J. El tema de nuestro tiempo (1923). En: Obras completas. Tomo III. Madrid: Alianza Editorial. Revista de occidente, 1983.

7. Marías J. Medicina para personas (y II). Jano 1987; 23: 1118.

8. Bergner M. Measurement of health status. Med Care 1985; 23: 696-704.

9. Kazis LE, Anderson JJ, Meelan RF. Effect sizes for interpreting changes in health status. Med Care 1989; 27: 178-89.

10. Patrick DL, Deyo RA. Generic and disease-specific measures in assessing health status and quality of life. Med Care 1989; 27: 217-32.

11. Siegrist J, Broer M, Junge A. Perfil de calidad de vida en enfermos crónicos (PECVEC). Versión oficial española del Profil der Lebensqualität Chronischkranker (PLC): Servicio Publicaciones de la Universidad de Oviedo, 1997.

12. Fernández-López JA, Siegrist J, Hernández-Mejía R, Broer M, CuetoEspinar A. Evaluación de la equivalencia transcultural de la versión española del Perfil de calidad de vida para enfermos crónicos (PECVEC). Med Clin 1997; 109: 245-50.

13. Fitzpatrick R. Assessment of quality of life as an outcome: finding measurements that reflect individual's priorities. Q Health Care 1999; 8: 1-

14. Ogihara T, Kuramoto K, Effect of long-term treatment with antihypertensive drugs on quality of life of ederly patients with hypertension: a double-blind comparative study between a calcium antagonist and a diuretic. NICS-EH Study group. National Intervention Corporative Study in Ederly Hypertensives. Hypertens Res 2000; 23: 33-7.

15. Crog SH, Levine S, Testa MA, Brown B, Bulpitt CJ, Jenkins CD. The effects of antihypertensive therapy on quality of life in clinical medicine. Soc Sci Med 1986; 314: 1657-64.

16. Fernández-López JA, Siegrist J, Hernández-Mejía R, Broer M, CuetoEspinar A. Study of quality of life on rural hypertensive patients. Comparison with the general population of the same environment. J Clin Epidemiol 1994; 47: 1373-80.

17. Bansal S. Sexual dysfunction in hypertensive men. A critical review of the literature. Hypertension 1988; 12: 1-10.
18. Beto JA, Bansal VK. Quality of life in treatment of hypertension. A metaanalysis of clinical trials. Am J Hypertens 1992; 5: 125-33

19. Di Somma S, Liguori V, Petitto M, Cavallotti G, Savonitto S, de Divitis O. Hemodynamic interactions between diuretics and calcium antagonists in the treatment of hypertensive patients. Cardiovasc Drug Ther 1990; 4: 1151-6.

20. Morgan TO, Anderson A, Jones E. Comparison and interaction of low dose felodipine and enalapril in the treatment of essential hypertension in ederly subjets. Am J Hypertens 1992; 5: 238-43.

21. Bühler FR. The case for calcium antagonist as first-line treatment of hypertension. J Hypertens 1990; 10: S17-S20.

22. Kelly JG, O’Malley K. Clinical pharmacokinetics of calcium antagonist. Clin Pharmacokinet 1992; 22: 416-33.

23. Parmley WW. New calcium antagonist: relevance of vasoselectivity. Am Heart J 1990; 120: 1408-13.

24. Hansson L. The Hypertension Optimal Treatment study and the importance of lowering blood pressure. J Hypertens (Supl.) 1999; 17: S9-13.

25. Staessen JA, Fagard R, Thijs L, Celis H, Birkenhager WH, de Leeuw $\mathrm{PW}$, et al. Subgroup and perprotocol analysis of the randomised European Trial on Isolated Systolic Hypertension in the Elderly. Arch Intern Med 1998; 158: 1681-91.

26. Control de la hipertensión arterial en España, 1996. Ministerio de Sanidad y Consumo. Sociedad-Liga Española para la Lucha contra la Hipertensión Arterial. Madrid 1996.

27. Spielberger CD, Gorsuch RL, Lushene RE. Cuestionario de ansiedad estado-rasgo. TEA Ediciones. Madrid. 1988.

28. Abellán J, García Teruel MJ, Abellán AF, García Muñoz M, Menárguez FH, Ferrer JM, et al. Implicaciones psicológicas de la observancia al tratamiento antihipertensivo. Hipertensión 1994; 11:3-8.

29. Martín Zurro A, Cano Pérez JF. Atención Primaria, conceptos, organización y práctica clínica. $3^{\text {a }}$ Ed. Doyma libros 1994; 30: 463-4.

30. Izzo JL, Levy D, Black HR. Clinical Advisory Statement: impotance of systolic blood pressure in older Americans. Hypertensyon 2000; 35: 1021-4.

31. Primatesta P, Brooke M, Poulter NR, Improved hypertension management and control. Result from the health survey for England 1998. Hypertension 2001; 38: 827-32.

32. Najman JM, Levine S. Evaluating the impact of medical care and technologies on the quality of life: A review and critique. Soc Sci Med 1981; 15: 107-15.

33. Ovretveit J. Evaluating health interventions. Filadelfia: Open University Press, 1997.

34. Bowling A. Research methods in health. Filadelfia: Open University Press, 1997.

35. Townsend R, Dipeette DJ, Evans RR, Davis WR, Green A, Graham G A, Wallace JM, Holland OB. Effects of calcium channel blockade on calcium homeostasis in mild to moderate essential hypertension. Am $\mathbf{J}$ Med Sci 1990; 300: 133-7.

36. Badia X, Carné X. La evaluación de la calidad de vida en el contexto del ensayo clínico. Med Clin 1998; 110: 550-6. 